\title{
Wood Knot Classification using Bagging
}

\author{
S. Mohan \\ Assistant Professor \\ Department of CSE \\ Annamalai University
}

\author{
K. Venkatachalapathy \\ Associate Professor \\ Department of CSE \\ Annamalai University
}

\begin{abstract}
The quality of the wood is determined by the number of defects and its distribution. In a piece of timber, the most common type of imperfection is called knot that decreases the strength of the wood. Manual selection and classification process of knots is tedious and time consuming job. An automatic sensing machine is able to inspect wood automatically and correctly identify the defects it possess, and its effect on the quality of the final product. In this paper, it is proposed to detect and classify the knots in timber boards. The image of knots is pre processed using Hilbert transform and Gabor filters. The features obtained from pre processing, is classified using data mining techniques and compared with bagging technique.
\end{abstract}

\section{Index Terms}

Wood Knots, Hilbert Transforms, Gabor Filter, Naïve Bayes, Radial Basis Function

\section{INTRODUCTION}

Wood is an integral part of human life with the need of wood increasing with the development. Wood is irreplaceable by any other synthetic material due to its inherent characteristics. The quality of the wood is determined by the number of defects and its distribution. Blue stains, splits, knots, inclusions are some of the defects present in wood. Knots are the most common defect seen in wood. Knots decrease the strength of the wood. The shape, size, location and type of knot are significant in deciding the grade and structural integrity of the wood [1]. The timber is carefully inspected to remove the knots to improve the quality and value of wood. Manual selection and classification process of knots is tedious and time consuming job. An automatic sensing machine which is able to inspect wood automatically and correctly identify the defect it possesses, and its effect on the quality of the final product is of great value.

The cleavability, cracking and warping and ease in working of timber are materially affected by knots. The strength of the timber is weakened and subsequently the value for structural uses is reduced due to the defects in the timber. The defective timber when subjected to a force that is perpendicular to the tension and/or grain and in the under load besides the compression and/or grain tend to have weakening effect. The strength of a beam affected by the knots depends on its size, condition, position, direction of fibre and number. For instance, even a small knot at the corner decreases the structural performance significantly, whereas, knots near the center of the timber piece has only slight effect in the performance of the timber. The stiffness of the structural timber is not essentially affected by the knots but the elastic limit of beams is greatly affected. The stiffness and elastic strength predominantly depend on the quality of wood fibre. Knots reduce the difference between the fibre stress at elastic limit and the modulus of rupture of beams.

Knots are formed when branch starts to grow out of trunk and wood cells in the trunk bend around the branch. The most common type of knots are:

- Crack Knot: A knot with a crack structure inside it is called Crack knot. The wood strength and its appearance are affected due to this type of knot.

- Dead Knot: A fully dark knot is called Dead knot. These types of knot have slightly darker area and have similar features as sound knot.

- Edge Knot: A knot that are on the edge of the timber.

- Intergrown Knot: A knot that is enclosed with strong bark areas is called Intergrown knot. There is a thin ring in these knots and big bark pockets are also found in these knots.

- Sound Knot: A knot with no defects (i.e., clear knot) is called a Sound knot. This type of knot is commonly not harmful. There are few circular shaped curves that reflect the age of the branches that is observed in the images of typical sound knots. When compared to the background wood, sound knots have slightly dark colors and various textures but it is much brighter than dead knot.

Typical image exemplars of wood knots are used in this study are shown in Fig.1.The knots shown in the figure are dry knots, horn knots, edge knots and sound knots respectively. The knot images show difference in color, shape, texture and size as it is observed in the figure.

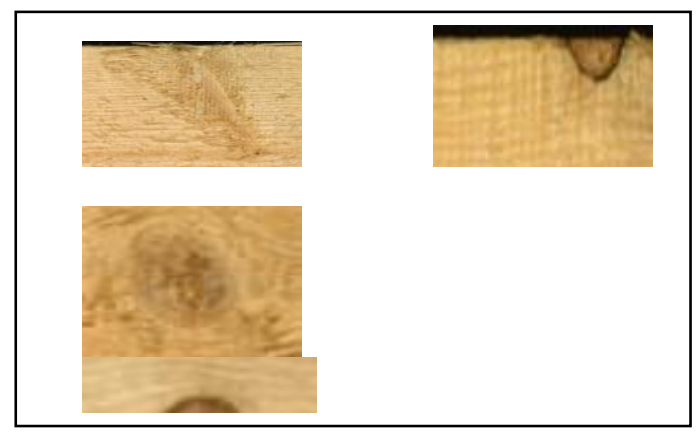

Fig. 1: Typical Examples of Knots

A sensing system is able to inspect wood automatically and correctly identifies the significant features it possess that affects the quality of the final product. The recognition and location of defects for both grading and removal purposes is performed correctly and continuously by this system. There is no need to perform $100 \%$ accuracy by this automatic 
inspection system but the vital role is to perform at or above the human standards constantly. To increase the higher value cuttings, the automated system is capable to identify the defects and also able to compute an optimal sawing approach. This type of system shows improved yield and decreased labor costs. The process of automation aspire to advance the speed of operation, increase the quality and thereby the productivity also. Hence, the novel system should be costeffective, time efficient, capable to perform in very high speed and the most significant thing is to have more accuracy level in detection and classification of knots.

It is not easy to develop a system for automatic defect classification of wood knots. Some aspects recognized for the complexity of problem in the basis of wood materials are:

- The texture on the surface of the wood

- The color of the surface of the wood

- The non-uniformity in surface of the wood

- The defect appears like a texture.

\section{RELATED WOrkS}

Mario I. Chacon [2] proposed to classify four kind of knot defect (encased knot, leaf knot, sound knot, edge knot) based on Gabor Filter. Gabor Filters were used to generate seven Gabor features to represent the standard deviation of images. Self Organizing Neural Network (SONN) and Feed Forward Perceptron Neural Networks are used as classifiers. The features selected were classified; the results show that both the classifiers perform better than human inspection of $85 \%$. SONN resulted an average classification rate $91.66 \%$ and $85.28 \%$ in testing, while FFPN with $97.22 \%$ in training and $91.17 \%$ in testing.

Irene $\mathrm{Gu}$, et al., [3] proposed a tree-structure Support Vector Machines (SVM) to classify five different wood knots type. The knots classified were Sound, Dead, Black, Rotten and Pin Knot from lumber boards. In the proposed method, the essential features were color, texture and size of knot. The knot area was divided into three regions for simplification of the process distribution of wood area. The three regions are: the interior area of knot (the knot area), the exterior area (a small background area) and the boundary area (the thick outer boundary of knot). Experimental results demonstrated the classification accuracy of the proposed method.

Stove [4] proposed Tree-Structured Support Vector Machine for wood knot classification. Tree structures SVM divide a large classifier into a few smaller and simpler ones thus reducing computational time. Pin knot has the smallest size when compared to other knots, the classifier using the size feature $\mathrm{X}$ distinguishes between pin knots and the remaining knots. Thus, the feature vector in the remaining levels of classifier would reduce. Similarly, dead knots have a prominent dark boundary curve against the interior and exterior area, the 2 level of classifier distinguishes black and non-black knots. This process is repeated for all classes, in the last level, "not recognized knot" type that do not belong to the four designed classes would not be classified. Experiment results show that the proposed SVM classifier achieved an average classification rate of $96.5 \%$ over 400 test knot images.

Pham, et al., [5] proposed a novel Bees algorithm to optimize a Support Vector Machine (SVM) for knot classification. The proposed algorithm is based on the foraging of food behavior of honey bees. The algorithm also selects the components constructing the feature vector that is to be contained in the SVM. This was performed in wood veneer sheets. To exploit the defect classification accuracy, the concern was to adapt the best combination of SVM parameters and data features which the proposed method obtained higher accuracy than that of the standard SVM.

Estevez, et al, [6] established a suitable set of characteristics (features) for the defect classification of radiate pine boards automatically. A genetic algorithm is employed in the proposed method. Using a low-cost machine vision system comprising a color video camera, a frame grabber, and a microcomputer was used. The following 10 defect types were: hole, live knot, pith, dead knot, blue stain, wane, split, stain, plus clear wood: birds eye $\&$ freckle and bark \& pitch pockets. A design of database that consists of color images of 2,958 board faces. From these images, totally 16,800 feature vectors were obtained and divided into test, training and validation sets. A total of 182 features were observed in each vector that was measured in the windows around the objects and the segmented objects. 64 from 182 original features were chosen and applied as inputs in a multilayer perceptron neural network classifier with the use of feature selection algorithm. This was performed with no decrease in the classification performance. The best off-line performance achieved was $74.5 \%$ of exact classifications in the test set when using a set of features developed by a genetic algorithm. $87.8 \%$ was the classification performance achieved in a reduced database involving 7 defect types. With 10 defect types plus clear wood, an online system calculation obtained $80 \%$ of correct classification. Our work proves that the more related features in complex classification problems like wood defect classification in which the best features are not recognized our genetic selection of features permits it to be recognized in an efficient way.

Zhen Hou et al., [7] examined a new technique to detect defects on the textured surfaces with the use of a Support Vector Machines (SVM) classification method along with the Gabor wavelet features. In order to decrease the computational cost on feature extraction, in performing a considerable detection rate an adaptive filter selection method is applied as substituting in the use of all filters in the Gabor wavelets. To organize a training data for a binary SVM classifier that performs a classification of pixel as defective or non-defective,an One-Against-All method is implemented. By comparing the experimental results with other multiresolution features and the Learning Vector Quantization (LVQ) classifier illustrated the efficiency of our proposed technique on the defect detection on textured surfaces. The classifiers trained including diverse proportions of data set showed the classification results that performed increasing as the proportion of the training data increased.

\section{METHODOLOGY}

This section gives the algorithm used for feature extraction, texture feature extraction and classifiers in the study.

\subsection{Hilbert Transforms}

The Hilbert transform [8] of a function $x(t)$ is given by

$h(t)=H\{x(t)\}=\frac{1}{\pi} \int^{+\infty} \frac{x(\tau)}{t-\tau} d(\tau)$

Using the Fourier identities, the Fourier transform of the Hilbert transform of $x(t)$ id

$h(t) \Leftrightarrow H(f)=-j \operatorname{sgn}(f) X(f)$

where $x(t) \Leftrightarrow X(f)$ is a Fourier transform pair and 
$\operatorname{sgn}(f)=\left\{\begin{array}{cl}1 & f>0 \\ 0 & f=0 \\ -1 & f<0\end{array}\right.$

\subsection{Gabor Filter}

Basically, Gabor filters are a wavelet group, where each wavelet captures energy at a specific frequency and specific direction. Expanding a signal with this gives a localized frequency description, and captures the signal's local features/energy. Texture features are then extracted for energy distributions. The scale (frequency) and orientation tunable property of Gabor filter makes it fit for texture analysis [9].

A two dimensional Gabor function $\mathrm{g}(\mathrm{x}, \mathrm{y})$ and its Fourier transform $\mathrm{G}(\mathrm{u}, \mathrm{v})$ is given by [10]:

$$
\begin{aligned}
& g(x, y)=\left(\frac{1}{2 \pi \sigma_{x} \sigma_{y}}\right) \exp \left[-\frac{1}{2}\left(\frac{x^{2}}{\sigma_{x}^{2}}+\frac{y^{2}}{\sigma_{y}^{2}}\right)+2 \pi j W x\right] \\
& G(u, v)=\exp \left\{-\frac{1}{2}\left[\frac{(u-W)^{2}}{\sigma_{u}^{2}}+\frac{v^{2}}{\sigma_{v}^{2}}\right]\right\}
\end{aligned}
$$

Where $\sigma_{u}=1 / 2 \pi \sigma_{x}$ and $\sigma_{v}=1 / 2 \pi \sigma_{y}$.

\subsection{Bagging}

Bagging was proposed by Leo Breiman in 1994 to improve classification accuracy by combining classifications from randomly generated training sets [11] as it incorporates models learned on different samplings for the original dataset. Bagging aims at variance reduction by building multiple models from samples of the training data. A committee of classifiers is formed by bootstrap aggregation of training sets from the original training dataset. Bagging replaces from the original training data by random sampling to create 'bags' for the committee of classifiers. Bagging improves performance over a single classifier created on all of the original data [11, $12,13]$

The bagging algorithm is as follows:

a. Generate bootstrap sample

$$
\left(X_{1}^{*}, Y_{1}^{*}\right), \ldots .,\left(X_{n}^{*}, Y_{n}^{*}\right)
$$

and compute the bootstrapped estimator $\hat{g}^{*}($.

b. Repeat step $1 \mathbf{B}$ times, forming

$$
\hat{g}^{* 1}(.), \ldots . ., \hat{g}^{* B}(.)
$$

c. Aggregate the bootstrap estimates

$$
\hat{g}_{\text {bag }}(.)=B^{-1} \sum_{i=1}^{B} \hat{g}^{*_{i}}(.)
$$

The bagging algorithm approximates,

$$
\hat{g}_{\text {Bag }}(.) \approx \mathrm{E}^{*}\left[\hat{g}^{*}(.)\right]
$$

\subsection{Naïve Bayes}

Naïve Bayes classifies the given input represented by its feature vector to the most likely class. Learning is simplified on the assumption that the features are independent given class,

$$
\begin{aligned}
& P(X \mid C)=\prod_{i=1}^{n} P\left(X_{i} \mid C\right) \\
& \text { Where } \mathrm{X}=\left(\mathrm{X}_{1}, \ldots, \mathrm{X}_{\mathrm{n}}\right) \text { is the feature vector and } \mathrm{C} \text { is a class. }
\end{aligned}
$$

\section{RESULTS AND DISCUSSION}

Evaluation was carried out using 400 images of wood knots. The types of knots chosen were dry knot, sound knot, horn knot and edge knot. The dataset consists of 100 images of each class of knot. The images are preprocessed using Hilbert transform for feature extraction and Gabor filter for feature reduction. Experiments are conducted using various classifiers like Naïve Bayes, Radial Basis Function. Bagging is used with Naïve Bayes, K Nearest Neighbor (KNN), REP trees and Random forest. Four approaches were used to creating a committee of classifiers, with each individual classifier created using $1 / 4^{\text {th }}$ of the original training dataset. Table 1 gives the classifier model parameters.

Table 1: Classification Accuracy and RMSE for different Classification Techniques

\begin{tabular}{|l|c|c|}
\hline \multicolumn{1}{|c|}{ Techniques } & $\begin{array}{c}\text { Classification } \\
\text { Accuracy(\%) }\end{array}$ & RMSE \\
\hline Naïve Bayes & 79 & 0.3207 \\
\hline Radial Basis Function & 81 & 0.3044 \\
\hline Bagging with REP tree & 61 & 0.3697 \\
\hline Bagging with Naïve Bayes & 80 & 0.2999 \\
\hline Bagging with Random forest & 81 & 0.342 \\
\hline Bagging with KNN & 72 & 0.3383 \\
\hline
\end{tabular}

The classification accuracy and Root Mean Square Error (RMSE) obtained from the proposed method using various classifiers is shown in Fig.2.

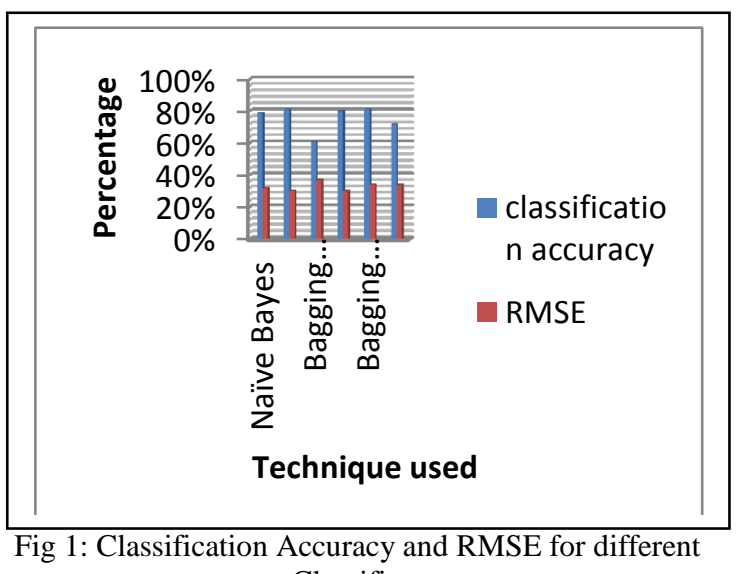
Classifiers

Table 2 tabulates precision and recall and f Measure. Fig.3 and Fig.4 shows the precision, recall and F-Measure. 
Table 2: Precision and Recall

\begin{tabular}{|l|c|c|c|}
\hline \multicolumn{1}{|c|}{ Techniques } & Precision & Recall & $\begin{array}{c}\text { F- } \\
\text { Measure }\end{array}$ \\
\hline Naïve Bayes & 0.791 & 0.79 & 0.79 \\
\hline Radial Basis Function & 0.811 & 0.81 & 0.81 \\
\hline Bagging with REP tree & 0.614 & 0.61 & 0.607 \\
\hline Bagging with Naïve Bayes & 0.76 & 0.792 & 0.921 \\
\hline $\begin{array}{l}\text { Bagging with Random } \\
\text { forest }\end{array}$ & 0.82 & 0.81 & 0.809 \\
\hline Bagging with KNN & 0.755 & 0.72 & 0.706 \\
\hline
\end{tabular}

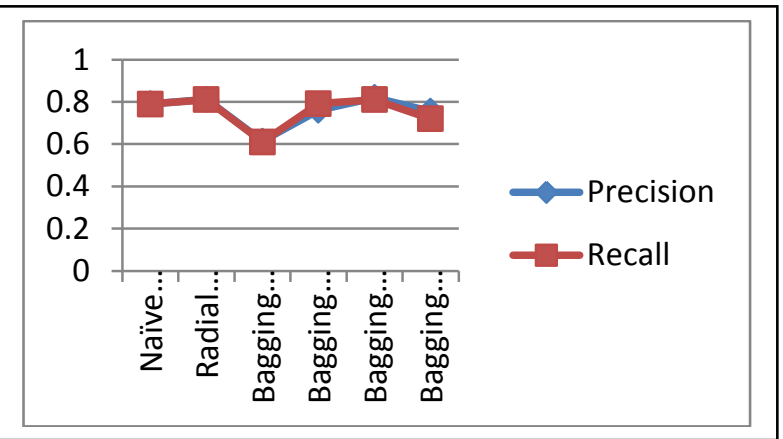

Fig.3: Precision and Recall

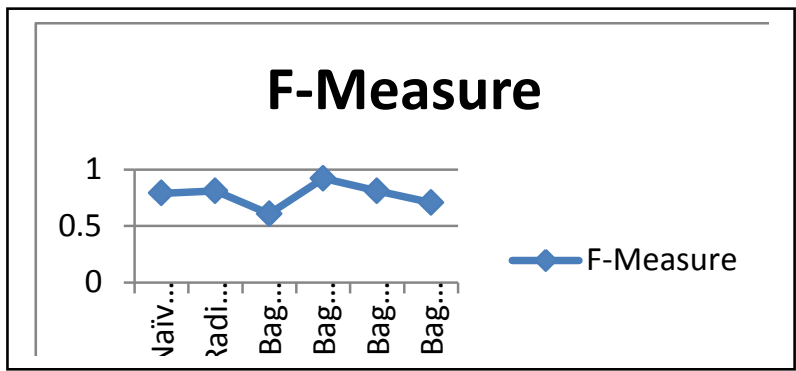

Fig 4: F- Measure for different Classifiers

\section{CONCLUSION}

In this paper, it was proposed to extract features from the images using Hilbert Transform and Gabor Filter for texture feature. Features extracted is classified using Naïve Bayes, Radial Basis function and Bagging Techniques. Results obtained by RBF shows high classification accuracy. Further experiments are conducted by bagging of dataset. The committee of classifiers used was Naïve Bayes, REP tree, Random forest and KNN. Future direction using soft computing techniques need to be investigated.

\section{REFERENCES}

[1] Brian Jr. Bond. Characterization of Wood Features Using Color, Shape, and Density Parameters. PhD thesis, 1998

[2] Mario I. Chacon and Graciela Ramirez Alonso. Wood defects classification using a som/ffp approach with minimum dimension feature vector. Volume 3973, pages $1105\{1110$, May 2006.

[3] Raul Vicen Irene Gu, Henrik Andersson. Wood defect classification based on image analysis and support vector machines. November 2009.

[4] A.G Stove. A doppler-based target classifier using linear discriminants and principal components. October 2004

[5] D. Pham, Z. Muhamad, M. Mahmuddin, A Ghanbarzadeh, E. Koc, and S. Otri, "Using the bees algorithm to optimise a support vector machine for wood defect classification," in Memorias del Innovative Production Machines and Systems Virtual Conference, 2007.

[6] Estévez, P.A., C.A. Perez, and E. Goles. 2003, Genetic input selection to a neural classifier for defect classification of radiata pine boards. Forest Prod. J. 53(7/8):87-94.

[7] Zhen Hou, Johne M. Parker "Texture Defect Detection Using Support Vector Machines with Adaptive Gabor Wavelet Features", "IEEE Workshop on Applications of Computer Vision”, Vol1, pp 275-280, 2005.

[8] Pei, S.-C.; Jaw, S.-B.; "Computation of discrete Hilbert transform through fast Hartley transform,"Circuits and Systems, IEEE Transactions on, vol.36, no.9, pp.12511252, Sep 1989.

[9] Dengsheng Zhang, Aylwin Wong, Maria Indrawan, Guojun Lu ,"Content-based Image Retrieval Using Gabor Texture Features

[10] C J Setchell, N W Campbell ,'Using Colour Gabor Texture Features For Scene Understanding." In Proc. $7^{\text {th }}$ Internat Conf. on image processing applications. Vol. 67(5), pp. 372-376

[11] Leo Breiman (1996). "Bagging predictors". Machine Learning 24 (2): 123-140. doi:10.1007/BF00058655.

[12] J. R. Quinlan. Bagging, boosting, and C4.5. In Proceedings of the Thirteenth National Conference on Artificial Intelligence, pages 725-730, 1996.

[13]E. Bauer and R. Kohavi. An empirical comparison of voting classification algorithms: Bagging, boosting and variants. Machine Learning, 36(1,2), 1999. 